PHYSICAL REVIEW A 95, 059902(E) (2017)

\title{
Publisher's Note: Continuous measurement of an atomic current [Phys. Rev. A 95, 043843 (2017)]
}

\author{
C. Laflamme, D. Yang, and P. Zoller
}

(Received 2 May 2017; published 9 May 2017)

DOI: 10.1103/PhysRevA.95.059902

This paper was published online on 28 April 2017 with errors in Eq. (D2). Equation (D2) should read as

$$
\begin{aligned}
d \hat{\rho}_{c}(t)= & -i\left[\hat{H}_{\text {atom }}, \hat{\rho}_{c}\right] d t+\frac{\gamma_{m}}{2}\left(2 \hat{c}_{m} \hat{\rho}_{c} \hat{c}_{m}^{\dagger}-\hat{c}_{m}^{\dagger} \hat{c}_{m} \hat{\rho}_{c}-\hat{\rho}_{c} \hat{c}_{m}^{\dagger} \hat{c}_{m}\right) d t \\
& +\sqrt{\gamma_{m}}\left[\left(\hat{c}_{m}-\left\langle\hat{c}_{m}\right\rangle_{c}\right) \hat{\rho}_{c}+\hat{\rho}_{c}\left(\hat{c}_{m}^{\dagger}-\left\langle\hat{c}_{m}^{\dagger}\right\rangle_{c}\right)\right] d W+\sum_{s} \frac{\gamma_{s}}{2}\left(2 \hat{c}_{s} \hat{\rho}_{c} \hat{c}_{s}^{\dagger}-\hat{c}_{s}^{\dagger} \hat{c}_{s} \hat{\rho}_{c}-\hat{\rho}_{c} \hat{c}_{s}^{\dagger} \hat{c}_{s}\right) d t
\end{aligned}
$$

The equation has been corrected as of 2 May 2017. The equation is correct in the printed version of the journal. 